\title{
A Secure Bio- Metric Fingerprint Recognition using Neural Network
}

\author{
Manpreet Kaur \\ M.Tech (Scholar), CSE \\ Punjabi University, Patiala
}

\author{
Sumandeep Kaur \\ Asst. Prof. (UCOE) \\ Punjabi University, Patiala
}

\begin{abstract}
Finger Print authentication system is one of the most common and often used bio metric analysis through which several organizations register the presence of their workers and employees. There are also some organizations in which verification of user is so critical that un-authorized access may harm the organization to a great level. So, this research work presents a unique verification system which is called finger print biometric authentication using Back Propagation Neural Network method. Mat lab 2010a version is being used to develop an algorithm. This technique can be made more reliable by using GA optimization method.
\end{abstract}

\section{Keywords}

Neural Network, Genetic Algorithm, Fingerprint Verification.

\section{INTRODUCTION}

Fingerprint matching is one of the oldest forms of the biometric technologies that are being used so widely. In the field of criminal investigation, civilian, government and commercial devices applications such as passport, licence card, security device etc., use of fingerprint technology is employed. Fingerprints of the human being are unique and they can never be the identical. Fingerprint identification is the practice of identification of personality based on the impression pattern of ridges and valley on fingers. There is a widespread use of fingerprint technology yet only modest work has been done to know the distinctiveness of the fingers. There are many factors that led to the interruption of recognition of fingerprints like pressure, small pressing spot, atmosphere factors, device noise, skin suppleness etc. Fingerprints are unalterable and they can diminish only after death. From the Egyptian time, fingerprints were used as a signature. The main features of a biometric gauge are:

- universality

- distinctiveness

- Durableness

- Collectability

- $\quad$ Satisfactoriness

- Recital

A lot of previous work has been already been done in biometric recognition by lot of other research workers in which one of the recent work presents the finger print recognition problem through Genetic Algorithm and BPNN network for classification. But the main issue of all authentication systems is that it must have, high accuracy rate and low error rate. That's why proposed method deals with the feature extraction process along with some pre-processing and post processing steps. To develop an algorithm that can accurately recognize the fingerprints and then matching of fingerprints should be done effectively. The evaluation parameters will be as follows:
1. FAR
2. FRR
3. Accuracy

The current problem statement involves the implementation of Genetic Algorithm method for the classification of the finger print mechanism and classification will be done using Back propagation Neural Network. The aim is to develop a higher matching accuracy algorithm to achieve the high reliability of this method.

\subsection{Minutia Extraction}

This technique used in wide area, being the basis of the fingerprint comparison made by fingerprint examiners. Minutiae are extracted from the two fingerprints and stored as sets of points in the 2-d plane. Matching that is based on minutiae essentially consists of finding the alignment between the template and the input minutiae sets that results in the maximum number of minutiae pairings

Combination of patterns called ridges and valley develop the fingerprints. Single arched section is known the ridges whereas part between two adjoining ridges is known as valley and ridge termination is known as minutiae. For fingerprint matching mainly two features of minutiae are used 1 . Ridge ending 2. Ridge bifurcation. Fingerprint matching is very crucial and essential step in biometric technology. Fingerprint matching based on minutiae method is a very popular approach [2].

\subsection{Genetic Algorithm}

Genetic Algorithm is an evolutionary based algorithm for feature reduction features. Below is the methodology for application of genetic algorithm.

- At random, produce an initial population $\mathrm{M}(0)$.

- Compute as well as help save the actual fitness $\mathrm{f}(\mathrm{m})$ for every specific individual $\mathrm{m}$ in the current population $\mathrm{M}(\mathrm{t})$;

- Specify selection probabilities $\mathrm{p}(\mathrm{m})$ for every specific individual $m$ throughout $M(t)$ making sure that $\mathrm{p}(\mathrm{m})$ is actually proportional to $\mathrm{f}(\mathrm{m})$.

- Crank out $\mathrm{M}(\mathrm{t}+1)$ by simply probabilistically choosing individuals from $\mathrm{M}$ ( $\mathrm{t}$ ) to produce offspring via genetic operators.

- Repeat step 2 until satisfying solution is actually attained.

\subsection{Neural Network}

Neural networks are those networks that are the collection of 
simple elements which function parallel. A neural network can be trained to perform a particular function by adjusting the values of the weights between elements. Network function is determined by the connections between elements. There are several activation functions that are used to produce relevant output.

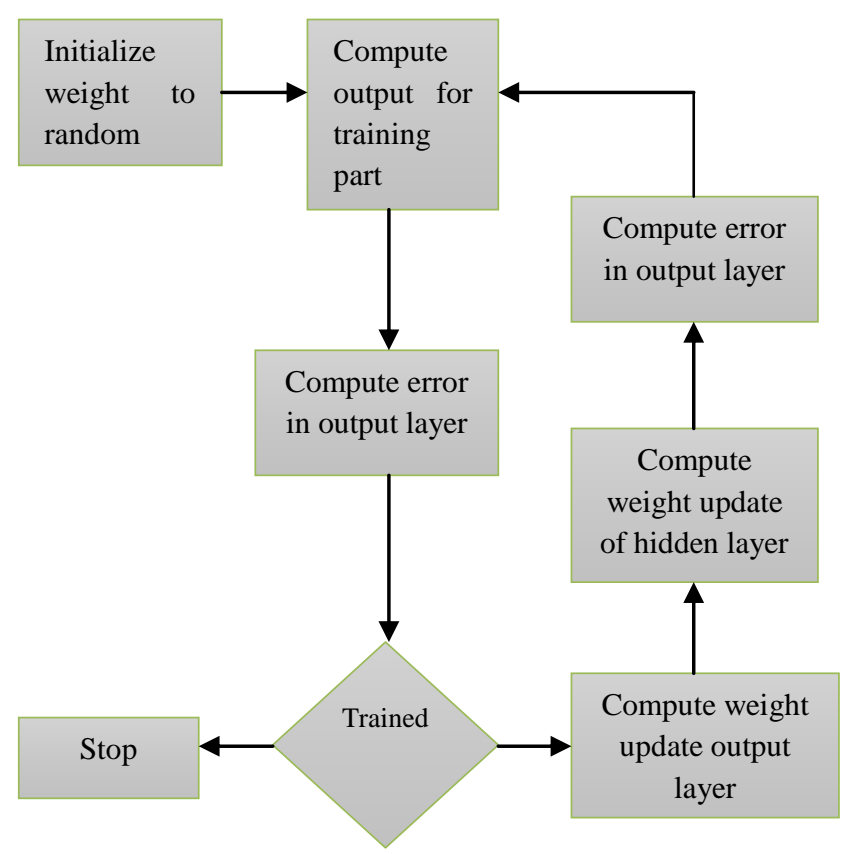

Fig 1: Neural Network

\section{PROPOSED WORK}

The more popular and widely used techniques, minutiae-based methods, use a feature vector extracted from the fingerprints and stored as sets of points in the multi-dimensional plane. The feature vector may contain minutiae's positions, orientations, types or all of them, etc. It essentially consists of finding the best alignment between the template and the input minutiae sets. However, minutiae-based methods may not utilize the rich discriminatory information available in the fingerprints with a high computational complexity. The texture-based methods use different types of features from the fingerprint ridge patterns such as local orientation and frequency, ridge shape and texture information. The features may be extracted more reliably than minutiae. Among various texture based matching methods, Gabor feature-based method and its improved version show relatively high performance comparing to previous works.

The proposed methodology will follow following steps:

\subsection{Fingerprint Acquisition}

It has been done from digital camera. The size of image must be less than $50 \mathrm{~kb}$ so that preprocessing can be done effectively and in less time. In proposed work tif format images has been chosen.

\subsection{Binarization}

It is the initial step of fingerprint matching. It is done to highlight the foreground pattern from the background. It is the process of conversion of grayscale image into binary image. Two values has been used in binarization i.e. 1 and 0.1 value is given to the foreground pattern so that its visibility can be increased whereas 0 value is given to background to make the visibility zero.

\subsection{Apply Minutia Extraction method}

Minutia matching is one of the very popular method in the field of fingerprint matching. In this method, minutiae points of one's fingers are matched with the other's minutiae points. Basically there are 80 minutiae points. Minutia based fingerprint matching involves minutia has the following features:

a) Form of minutia ( ridge ending or ridge bifurcation),

b) Location of minutia on the fingerprint image casing,

c) Angle with which ridge is allied with minutia makes the parallel axis.

d) Minutia direction

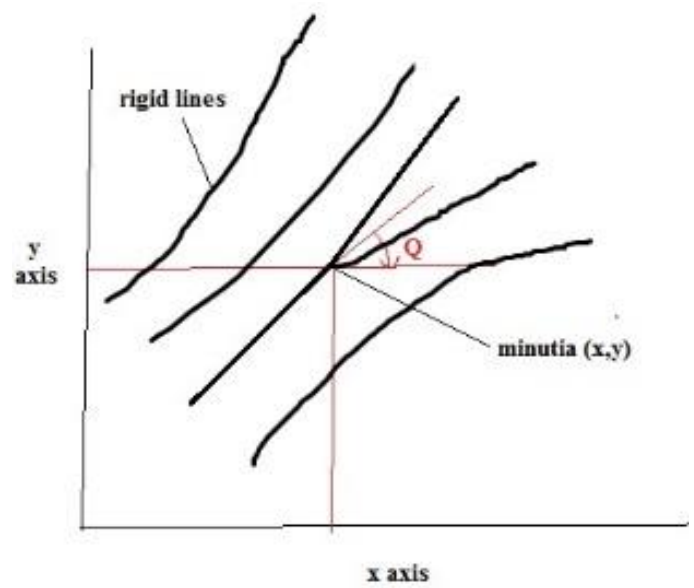

Fig 2: Minutia Structures

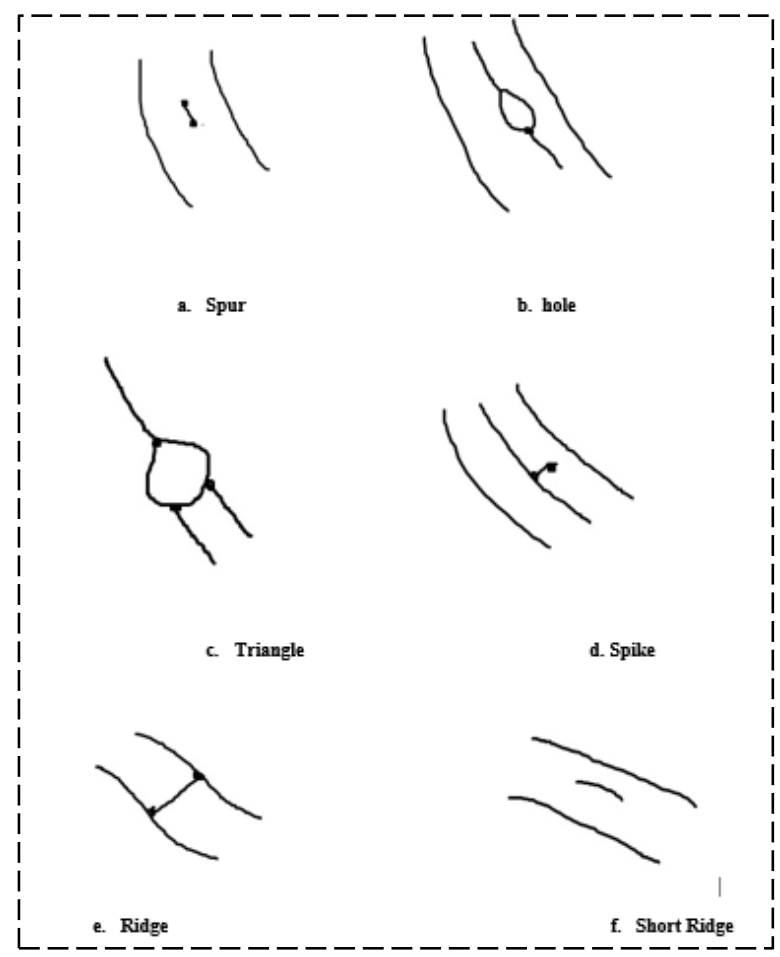

Fig 3: Minutia Characteristics

Two fingerprint impressions are matched by comparing minutia matching points. Examples of false minutiae structures are as following: 
- Spurs: a cut protrude from an edge

- Hole: hole is an elliptical arrangement occurs due to pores

- Triangle: matching is based on triangle type and triangle direction

- $\quad$ Spike: false correlation of two ridges

Removing of false minutia is a very important step of the preprocessing. Presence of large number of false minutia will led to the wrong fingerprint matching. False minutia removal has been taken place distinctively if following order to get the accuracy.

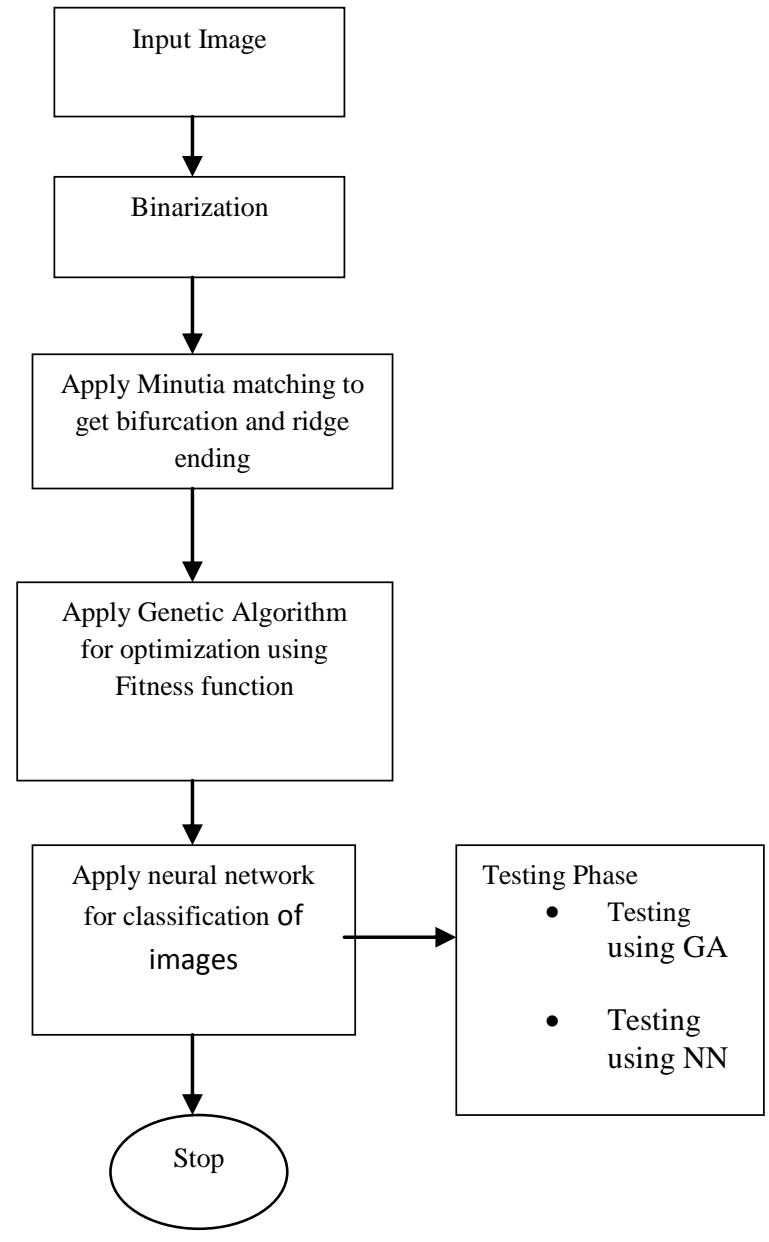

Fig 4: Proposed Model

\subsection{Apply Genetic Algorithm}

Now application of Genetic Algorithm has been done for optimization of features obtained from minutia extraction using below steps:

Fs=data_ga(k,i);Ft=mean(data_ga);

FitnessFunction = @ (e)fitness_fn $(\mathrm{e}, \mathrm{Fs}, \mathrm{Ft})$;

numberOfVariables $=1$;

[x(i)

fval]

ga(FitnessFunction,numberOfVariables,[],[],[],[],[],[],[],optio ns);

if $\operatorname{round}(x(i))>0$

Gareduced1 $(\mathrm{k}, \mathrm{p})=(\mathrm{x}(\mathrm{i}))$; $\mathrm{p}=\mathrm{p}+1$;

end

\subsection{Apply Neural Network for Classification}

Instead of using the threshold as the judgment rule, another method to measure the similarity between the feature vectors of the input fingerprint with those of the templates fingerprint stored in the database is realized by a three-layer BPNN. The structure of the BPNN consists of input layer, one hidden layer and output layer. Train Set function has been used for testing.

\section{RESULTS AND DISCUSSION 3.1 Metrics}

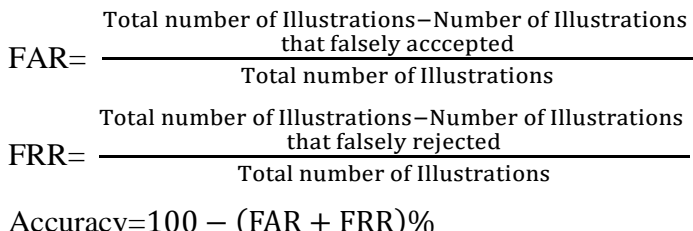

\subsection{Analysis}

Below table shows the values of FRR, FAR and Accuracy using proposed algorithm for various number of images. From simulation results average values of FRR $=.9998, \mathrm{FRR}=$ .07997 and Accuracy $=99.82$.

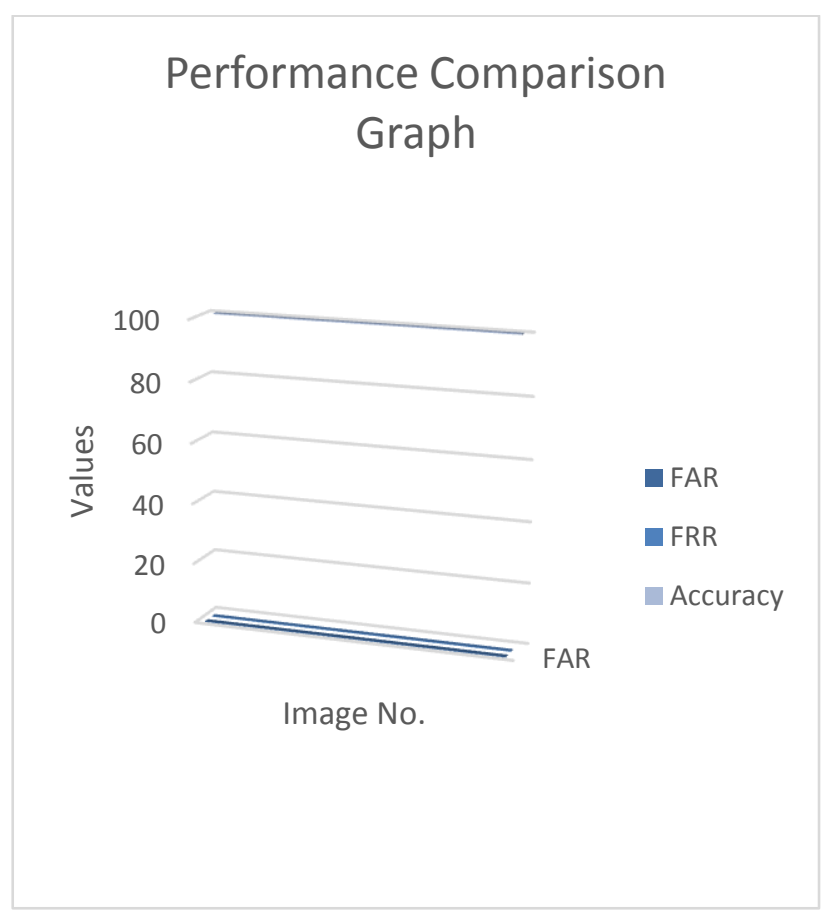

Fig 5: Comparison Graph 
Table 1: Parameter value w.r.t Image number

\begin{tabular}{|c|c|c|c|}
\hline $\begin{array}{l}\text { Image } \\
\text { Number }\end{array}$ & FAR & FRR & Accuracy \\
\hline 1. & .099984 & .079974 & 99.82 \\
\hline 2. & .099980 & .079969 & 99.83 \\
\hline 3. & .099979 & .079970 & 99.84 \\
\hline 4. & .099982 & .079971 & 99.85 \\
\hline 5. & .099981 & .079968 & 99.82 \\
\hline 6. & .099983 & .079976 & 99.81 \\
\hline 7. & .099985 & .079974 & 99.80 \\
\hline 8. & .099979 & .079973 & 99.83 \\
\hline 9. & .099980 & .079972 & 99.84 \\
\hline 10. & .099981 & .079970 & 99.84 \\
\hline 11. & .099984 & .079971 & 99.85 \\
\hline 12. & .099982 & .079969 & 99.86 \\
\hline 13. & .099983 & .079968 & 99.87 \\
\hline 14. & .099981 & .079974 & 99.82 \\
\hline 15. & .099980 & .079969 & 99.83 \\
\hline 16. & .099986 & .079974 & 99.84 \\
\hline 17. & .099983 & .079973 & 99.81 \\
\hline 18. & .099985 & .079972 & 99.80 \\
\hline 19. & .099982 & .079975 & 99.84 \\
\hline
\end{tabular}

\section{CONCLUSION AND FUTURE SCOPE}

In this work it has been shown that if a fingerprint image of a person is given then the network can recognize the image. The whole work is completed through the following steps:

1. 19 fingerprint images have been used for the experiment. Fingerprint images of various persons have been collected by taking different samples of each person.

2. Each image is converted into grayscale conversion to get the edges of the image.

3. Feed Forward Back Propagation neural network, Genetic Algorithm have been used to train, test and validate the network for each part of the image.

4. Output of all four are collected and set as the new input and a new target is set to train, test and validate the entire parts of each sample.

5. Fingerprint image without dividing into parts have also been applied in the network.

When the fingerprint image was divided into two parts to identify a person then it took 6-epoch while when the image was not decomposed then the average number of iterations were 8. Image division in this work has been done by using a matlab tool. Future work could be to develop a tool to automate some of the processes which have been done manually here.

Place Tables/Figures/Images in text as close to the reference as possible (see Figure 1). It may extend across both columns to a maximum width of $17.78 \mathrm{~cm}$ (7”).

Captions should be Times New Roman 9-point bold. They should be numbered (e.g., "Table 1" or "Figure 2"), please note that the word for Table and Figure are spelled out. Figure's captions should be centered beneath the image or picture, and Table captions should be centered above the table body.

\section{REFERENCES}

[1] Hartwing Fronthaler, Klaus kollreider, and Josef Bigun, "Local Features for Enhancement and Minutiae Extraction in Fingerprints", IEEE Transactions on Image Processing, vol. 17, no, 3, pp. 354- 363, (2008).

[2] M. R. Girgisa, A. A. Sewisyb and R. F. Mansourc, "Employing Generic Algorithms for Precise Fingerprint Matching Based on Line Extraction", Graphics, Vision and Image Procession Journal, vol. 7, pp. 51-59, (2007).

[3] Duresuoquian Miao, Qingshi Tang, and Wenjie Fu, "Fingerprint Minutiae Extraction Based on Principal Cures", the Journal of the Pattern Recognition Letters, vol. 28, pp. 2184-2189, (2007).

[4] Luping Ji, Zhang Yi, "Fingerprint Orientation field Estimation using Ridge Protection", The Journal of the Pattern Recognition, vol. 41, pp. 1491-1503, (2008).

[5] G.Sambasiva Rao, C. NagaRaju, L. S. S. Reddy and E. V. Prasad, "A Novel Fingerprints Identification System Based on the Edge Detection", International Journal of Computer Science and Network Security, vol. 8, pp. 394 397, (2008).

[6] Jinwei Gu, Jie Zhou, and Chunyu Yang, "Fingerprint Recognition by Combining Global Structure and Local Cues", IEEE Transactions on Image Processing, vol. 15, no. 7, pp. 1952 - 1964, (2006).

[7] V. Vijaya Kumari and N. Suriyanarayanan, "Performance Measure of Local Operators in Fingerprint Detection”, Academic Open Internet Journal, vol. 23, pp. $1-7,(2008)$

[8] Raju Sonavane and B. S. Sawant, "Noisy Fingerprint Image Enhancement Technique for Image Analysis: A Structure Similarity Measure Approach", Journal of Computer Science and Network Security, vol. 7 no. 9, pp. 225-230, (2007).

[9] Eric P. Kukula, Christine R. Blomeke, Shimon K. Modi, and Tephen J. Elliott, "Effect of Human Interaction on Fingerprint Matching Performance, Image Quality, and Minutiae Count", International Conference on Information Technology and Applications, pp. 771-776, (2008).

[10] Mana Tarjoman, and Shaghayegh Zarei, "Automatic Fingerprint Classification using Graph Theory", Proceedings of World Academy of Science, Engineering and Technology, vol. 30, pp. 831-835, (2008).

[11] Bhupesh Gour, T. K. Bandopadhyaya and Sudhir Sharma, "Fingerprint Feature Extraction using Midpoint Ridge Contour Method and Neural Network", International Journal of Computer Science and Network Security, vol. 8, no, 7, pp. 99-109, (2008). 